Article

\title{
A Note on Geodesic Vector Fields
}

\author{
Sharief Deshmukh ${ }^{1}\left(\mathbb{D}\right.$, Josef Mikeš ${ }^{2}\left(\mathbb{D}\right.$, Nasser Bin Turki ${ }^{1}\left(\mathbb{D}\right.$ and Gabriel-Eduard Vîlcu ${ }^{3, *(D)}$ \\ 1 Department of Mathematics, College of Science, King Saud University, P.O. Box 2455, \\ Riyadh 11451, Saudi Arabia; shariefd@ksu.edu.sa (S.D.); nassert@ksu.edu.sa (N.B.T.) \\ 2 Department of Algebra and Geometry, Palacky University, 17. Listopadu 12, \\ 77146 Olomouc, Czech Republic; josef.mikes@upol.cz \\ 3 Department of Cybernetics, Economic Informatics, Finance and Accountancy, Petroleum-Gas University \\ of Ploieşti, Bd. Bucureşti 39, Ploiești 100680, Romania \\ * Correspondence: gvilcu@upg-ploiesti.ro; Tel.: +40-244-575-847
}

Received: 14 August 2020; Accepted: 23 September 2020; Published: 27 September 2020

check for updates

\begin{abstract}
The concircularity property of vector fields implies the geodesicity property, while the converse of this statement is not true. The main objective of this note is to find conditions under which the concircularity and geodesicity properties of vector fields are equivalent. Moreover, it is shown that the geodesicity property of vector fields is also useful in characterizing not only spheres, but also Euclidean spaces.
\end{abstract}

Keywords: geodesic vector field; concircular vector field; $n$-sphere; Euclidean space

MSC: 53C20; 53C21; 53C24

\section{Introduction}

One of the themes of interest in Riemannian geometry concerns the presence of some vector fields of special type on Riemannian spaces, like (unit) geodesic vector fields (cf. [1-3]), Jacobi-type vector fields (cf. [4,5]), concircular vector fields (cf. [6-9]), torse forming vector fields (cf. [10-13]), Killing vector fields (cf. [14-20]), and conformal vector fields (cf. [21-24]). It is known that these vector fields of special type influence not only the geometry, but also the topology of the Riemannian spaces. In 1925, Brinkmann [25] studied conformal mappings between Einstein spaces, and he obtained Einstein metrics with concircular vector fields. Geodesic mappings of (pseudo-)Riemannian spaces with concircular vector fields were studied by many authors, the focus being on the case of geodesic mappings of Ricci flat, Kähler, semisymmetric, Ricci semi-symmetric, and product spaces (see, e.g., [26] and references therein). Moreover, analogous results for holomorphically projective mappings were obtained in [27-29].

Of all these distinguished vector fields outlined above, the least demanding on a Riemannian manifold $(M, g)$ is the geodesic vector field. This is a vector field $\mathbf{u}$ on $M$ characterized by the next differential equation

$$
\nabla_{\mathbf{u}} \mathbf{u}=\sigma \mathbf{u},
$$

where $\nabla_{\mathbf{u}}$ is the covariant derivative (with respect to the Levi-Civita connection) in the direction of $\mathbf{u}$, while $\sigma$ is a smooth function on $M$ called the potential function of $\mathbf{u}$. Moreover, if $\sigma=0$, then $\mathbf{u}$ is said to be a unit geodesic vector field (in this particular case, it is known that any integral curve is a geodesic). In the case of $\sigma \neq 0$, the geodesic vector field $\mathbf{u}$ is called a non-trivial geodesic vector field. It was pointed out earlier that the geodesicity property of a vector field demands a minimum of a Riemannian manifold on which it lives, and as a result, it is the most general type of special vector field, having a broad appearance in Riemannian geometry. For instance, every vector field with 
a concircularity property exhibits the geodesicity property, while Eikonal equations too give rise to geodesic vector fields (cf. [30]). As the definition of geodesic vector fields is very simple, the following question arises: Does the existence of non-trivial geodesic vector fields affect the geometry of a Riemannian manifold? In this paper, we answer this question ascertaining that yes, it does.

It is observed in Section 4 that there is a non-trivial geodesic vector field on $\mathbf{S}^{n}(c)$, the $n$-dimensional sphere of constant curvature $c$, that satisfies $\Delta \mathbf{u}=-c \mathbf{u}$, where $\Delta$ is the rough Laplacian operating on smooth vector fields of $\mathbf{S}^{n}(c)$. Then, it is demonstrated that this property, together with some restriction on the energy of a vector field with geodesicity property on a compact and connected Riemannian space gives a nice characterization for $\mathbf{S}^{n}(c)$ (cf. Theorem 2). Another characterization of the sphere $\mathbf{S}^{n}(c)$ using closed non-trivial geodesic vector fields is also given (cf. Theorem 3). Similarly, in Section 5, it is proven that every Euclidean space possesses a non-trivial geodesic vector field $\mathbf{u}$, satisfying $\Delta \mathbf{u}=0$, and also characterizes such a space using the geodesicity property of a harmonic vector field $\mathbf{u}$ and a restriction on the Laplacian of the length function of $\mathbf{u}$ (cf. Theorem 4). It was noticed that the concircularity property of vector fields involves the geodesicity property, but the converse of this assertion is not true. We provide examples of non-concircular geodesic vector fields in Section 3, and we also find suitable conditions under which the concircularity and geodesicity properties are equivalent (cf. Theorem 1). It is noteworthy that some distinct characterizations of the sphere and the Euclidean space in terms of the existence of non-trivial geodesic vector fields satisfying certain additional properties were recently obtained in [31], but these are of different nature and do not imply the characterizations established in the present work (cf. Remarks 1 and 2).

\section{Preliminaries}

Suppose $(M, g)$ is a Riemannian manifold of dimension $n$. Let us denote as usual by $\mathfrak{X}(M)$ the Lie algebra of smooth vector fields on $M$, and by $\nabla$ the Levi-Civita connection on $(M, g)$. The curvature tensor field $R$ on $(M, g)$ is defined by

$$
R(X, Y) Z=\nabla_{X} \nabla_{Y} Z-\nabla_{Y} \nabla_{X} Z-\nabla_{[X, Y]} Z, \quad X, Y, Z \in \mathfrak{X}(M),
$$

while the Ricci tensor Ric is given by

$$
\operatorname{Ric}(X, Y)=\sum_{i=1}^{n} g\left(R\left(e_{i}, X\right) Y, e_{i}\right),
$$

where $\left\{e_{1}, \ldots, e_{n}\right\}$ is a local orthonormal frame on $M$.

The Ricci operator $Q: \mathfrak{X}(M) \rightarrow \mathfrak{X}(M)$ is defined by (cf. [32])

$$
g(Q X, Y)=\operatorname{Ric}(X, Y), \quad X, Y \in \mathfrak{X}(M) .
$$

It is easy to see that $Q$ is a symmetric operator. On the other hand, the trace of the Ricci operator $Q$ is the scalar curvature of $(M, g)$, and it is denoted by $\tau$. Hence, $\tau=\operatorname{Tr} Q$. Given a smooth function $f: M \rightarrow R$, the Hessian operator $A_{f}$ is defined by

$$
A_{f} X=\nabla_{X} \nabla f, \quad X \in \mathfrak{X}(M),
$$

where $\nabla f$ stands for the gradient of $f$. The Hessian of $f$, denoted by Hess $(f)$, is defined by

$$
\operatorname{Hess}(f)(X, Y)=g\left(A_{f} X, Y\right), X, Y \in \mathfrak{X}(M),
$$


and the Laplacian $\Delta f$ of $f$ is defined as $\Delta f=\operatorname{Tr} A_{f}$, that is, the trace of the Hessian operator $A_{f}$. Given a Riemannian manifold $(M, g)$, the rough Laplacian $\Delta: \mathfrak{X}(M) \rightarrow \mathfrak{X}(M)$ is an operator that acts on smooth vector fields, given by

$$
\Delta X=\sum_{i=1}^{n}\left(\nabla_{e_{i}} \nabla_{e_{i}} X-\nabla_{\nabla_{e_{i}} e_{i}} X\right), \quad X \in \mathfrak{X}(M),
$$

where $\left\{e_{1}, \ldots, e_{n}\right\}$ is a local orthonormal frame on $M$ and $n=\operatorname{dim} M$. Recall that a smooth vector field $\xi$ on $M$ is said to be an eigenvector of the operator $\Delta$ if $\Delta \xi=-\lambda \xi$, where $\lambda \geq 0$ is a real constant. If $\lambda=0$, then $\xi$ is said to be a harmonic vector field.

A smooth vector field $\mathbf{v}$ on a Riemannian manifold $(M, g)$ is called a unit geodesic vector field if

$$
\nabla_{\mathbf{v}} \mathbf{v}=0
$$

that is, the integral curves of $\mathbf{v}$ are geodesics. On the other hand, a smooth vector field $\mathbf{v}$ on $M$ is said to be a geodesic vector field if

$$
\nabla_{\mathbf{v}} \mathbf{v}=\sigma \mathbf{v},
$$

where $\sigma$ is a smooth function on $M$, called the potential function of $\mathbf{v}$. Moreover, if $\sigma \neq 0$, then $\mathbf{v}$ is said to be a non-trivial geodesic vector field.

Recall now that a smooth vector field $\mathbf{v}$ on a Riemannian manifold $(M, g)$ is said to be a concircular vector field if

$$
\nabla_{X} \mathbf{v}=\sigma X, \quad X \in \mathfrak{X}(M),
$$

where $\sigma$ is a smooth function on $M$. In other words, the local flow of the concircular vector field consists of concircular transformations (cf. [7]). It follows from Equations (1) and (2) that a concircular vector field is a geodesic vector field.

Let $\mathbf{v}$ be a geodesic vector field on a Riemannian manifold $(M, g)$, and suppose $\alpha$ is the smooth 1 -form dual to the vector field $\mathbf{v}$, that is, $\alpha(X)=g(\mathbf{v}, X), X \in \mathfrak{X}(M)$. Now, let us define a symmetric operator $A: \mathfrak{X}(M) \rightarrow \mathfrak{X}(M)$ and a skew symmetric operator $\varphi: \mathfrak{X}(M) \rightarrow \mathfrak{X}(M)$ by

$$
\frac{1}{2}\left(\ell_{\mathbf{v}} g\right)(X, Y)=g(A X, Y), \quad \frac{1}{2} d \alpha(X, Y)=g(\varphi X, Y), \quad X, Y \in \mathfrak{X}(M),
$$

where $£_{\mathbf{v}} g$ stands for the Lie derivative of $g$ with respect to $\mathbf{v}$. Then, it follows that

$$
\nabla_{X} \mathbf{v}=A X+\varphi X, \quad X \in \mathfrak{X}(M) .
$$

For a $(1,1)$ tensor field $T$ on $M$, one can consider the covariant derivative $\nabla T$, defined as

$$
(\nabla T)(X, Y)=\nabla_{X} T Y-T\left(\nabla_{X} Y\right), \quad X, Y \in \mathfrak{X}(M) .
$$

Using Equation (4), we compute the following equation for curvature tensor

$$
R(X, Y) \mathbf{v}=(\nabla A)(X, Y)-(\nabla A)(Y, X)+(\nabla \varphi)(X, Y)-(\nabla \varphi)(Y, X)
$$

which gives

$$
\operatorname{Ric}(Y, \mathbf{v})=g\left(Y, \sum_{i=1}^{n}(\nabla A)\left(e_{i}, e_{i}\right)\right)-Y(s)-g\left(Y, \sum_{i=1}^{n}(\nabla \varphi)\left(e_{i}, e_{i}\right)\right)
$$


for a local orthonormal frame $\left\{e_{1}, \ldots, e_{n}\right\}$, where $s=\operatorname{Tr} A$ and we used the symmetry of $A$, and also the skew symmetry of $\varphi$. The above equation yields

$$
\operatorname{Ric}(\mathbf{v}, \mathbf{v})=g\left(\mathbf{v}, \sum_{i=1}^{n}(\nabla A)\left(e_{i}, e_{i}\right)\right)-\mathbf{v}(s)-g\left(\mathbf{v}, \sum_{i=1}^{n}(\nabla \varphi)\left(e_{i}, e_{i}\right)\right) .
$$

\section{A Characterization of Concircular Vector Fields}

As seen earlier, the concircularity property of vector fields implies the geodesicity property, but the converse in not true. We give some examples below to prove this.

(i) Consider the warped product $M=R \times{ }_{e^{t}} F$, where $\left(F, g_{F}\right)$ is a Riemannian manifold and $t$ is a coordinate function on the real line $R$ (cf. [33]). Let us denote by $g_{M}=d t^{2}+e^{2 t} g_{F}$ the warped product metric on $M$. Then, the vector field

$$
\mathbf{u}=e^{h} \frac{\partial}{\partial t^{\prime}}
$$

where $h: F \rightarrow R$ is a smooth function, provides us an example of a geodesic vector field that is not a concircular vector field. Indeed, by taking $X=f \frac{\partial}{\partial t}+U \in \mathfrak{X}(M)$, where $f: R \rightarrow R$ is a smooth function and $U \in \mathfrak{X}(F)$, then using [33] Proposition 35 for the Riemannian manifold $\left(M, g_{M}\right)$, we get

$$
\begin{aligned}
\nabla_{X} \mathbf{u} & =e^{h}\left(f \frac{\partial}{\partial t}+U\right)+e^{h} U(h) \frac{\partial}{\partial t} \\
& =e^{h} X+e^{h} U(h) \frac{\partial}{\partial t} .
\end{aligned}
$$

Thus, $\mathbf{u}$ is not a concircular vector field. However, the above equation gives

$$
\nabla_{\mathbf{u}} \mathbf{u}=e^{h} \mathbf{u},
$$

that is, $\mathbf{u}$ is a non-trivial geodesic vector field.

(ii) Consider the Euclidean space $\mathbf{E}^{n}$ with position vector field

$$
\Psi=\sum_{i=1}^{n} u^{i} \frac{\partial}{\partial u^{i}}
$$

where $u^{1}, \ldots, u^{n}$ are Euclidean coordinates. Let $f$ be a smooth function on $\mathbf{E}^{n}$, defined by

$$
f=\left(u^{1}\right)^{2}+\left(u^{2}\right)^{2}+\ldots+\left(u^{n}\right)^{2} .
$$

Then, the vector field $\xi=f \Psi$ satisfies

$$
\nabla_{\xi} \xi=3 f \xi,
$$

that is, $\xi$ is a non-trivial geodesic vector field, where $\nabla$ is the Euclidean connection on $\mathbf{E}^{n}$. It is clear that for any smooth vector field $X$ on $\mathbf{E}^{n}$, we have

$$
\nabla_{X} \xi=f X+X(f) \Psi
$$

and as

$$
X(f) \Psi \neq 2 f X,
$$

we conclude $\xi$ is not a concircular vector field.

Considering the above examples, it is a natural problem to find conditions under which the concircularity and geodesicity properties are equivalent. The aim of this section is to investigate this problem. It is quite fascinating to note that a suitable lower bound for the Ricci curvature Ric in the 
direction of a non-trivial geodesic vector field on a Riemannian manifold will give a necessary and sufficient condition for the equivalence of concircularity and geodesicity properties, provided that the Riemannian manifold is compact and connected.

Theorem 1. Let $\mathbf{v}$ be a non-trivial geodesic vector field on an $n$-dimensional compact and connected Riemannian manifold $(M, g)$. Then, $\mathbf{v}$ is concircular if, and only if the Ricci curvature Ric $(\mathbf{v}, \mathbf{v})$ satisfies

$$
\operatorname{Ric}(\mathbf{v}, \mathbf{v}) \geq \frac{1}{4}\|d \alpha\|^{2}-\frac{n-1}{2 n} \mathbf{v}\left(\operatorname{Tr} \xi_{\mathbf{v}} g\right),
$$

where $\alpha$ is the 1-form dual to $\mathbf{v}$ and $E_{\mathbf{v}} g$ stands for the Lie derivative of $g$ with respect to $\mathbf{v}$.

Proof. Let $\mathbf{v}$ be a non-trivial geodesic vector field that satisfies the given condition. Letting $s=\operatorname{Tr} A$ and using Equation (3), we see that the condition in the statement reads

$$
\operatorname{Ric}(\mathbf{v}, \mathbf{v}) \geq\|\varphi\|^{2}-\frac{n-1}{n} \mathbf{v}(s) .
$$

Using Equation (4) and also the symmetry of $A$, we find

$$
\operatorname{div} A \mathbf{v}=\|A\|^{2}+g\left(\mathbf{v}, \sum_{i=1}^{n}(\nabla A)\left(e_{i}, e_{i}\right)\right)
$$

and similarly using skew symmetry of the operator $\varphi$, we compute

$$
\operatorname{div} \varphi \mathbf{v}=-\|\varphi\|^{2}-g\left(\mathbf{v}, \sum_{i=1}^{n}(\nabla \varphi)\left(e_{i}, e_{i}\right)\right)
$$

where

$$
\|A\|^{2}=\sum_{i=1}^{n} g\left(A e_{i}, A e_{i}\right), \quad\|\varphi\|^{2}=\sum_{i=1}^{n} g\left(\varphi e_{i}, \varphi e_{i}\right) .
$$

Also, Equation (4) gives divv $=s$, and we have

$$
\operatorname{div} s \mathbf{v}=\mathbf{v}(s)+s^{2} .
$$

Now, using Equations (8) and (10), we conclude

$$
\|A\|^{2}-\frac{1}{n} s^{2}=\operatorname{div}\left(A \mathbf{v}-\frac{1}{n} s \mathbf{v}\right)-g\left(\mathbf{v}, \sum_{i=1}^{n}(\nabla A)\left(e_{i}, e_{i}\right)\right)+\frac{1}{n} \mathbf{v}(s) .
$$

Combining above equation with Equation (6), we get

$$
\|A\|^{2}-\frac{1}{n} s^{2}=\operatorname{div}\left(A \mathbf{v}-\frac{1}{n} s \mathbf{v}\right)-\operatorname{Ric}(\mathbf{v}, \mathbf{v})-g\left(\mathbf{v}, \sum_{i=1}^{n}(\nabla \varphi)\left(e_{i}, e_{i}\right)\right)-\frac{n-1}{n} \mathbf{v}(s),
$$

which on using Equation (9), gives

$$
\|A\|^{2}-\frac{1}{n} s^{2}=\operatorname{div}\left(A \mathbf{v}+\varphi \mathbf{v}-\frac{1}{n} s \mathbf{v}\right)+\|\varphi\|^{2}-\operatorname{Ric}(\mathbf{v}, \mathbf{v})-\frac{n-1}{n} \mathbf{v}(s) .
$$

Integrating above equation, we conclude

$$
\int_{M}\left(\|A\|^{2}-\frac{1}{n} s^{2}\right)=\int_{M}\left(\|\varphi\|^{2}-\frac{n-1}{n} \mathbf{v}(s)-\operatorname{Ric}(\mathbf{v}, \mathbf{v})\right),
$$


which, in view of Schwartz's inequality $\|A\|^{2} \geq \frac{1}{n} s^{2}$ and inequality (7), implies

$$
A=\frac{s}{n} I
$$

Using Equations (2), (4) and (12), we derive

$$
\varphi \mathbf{v}=\left(\sigma-\frac{s}{n}\right) \mathbf{v},
$$

and taking the inner product with $\mathbf{v}$ in above equation, we arrive at

$$
\left(\sigma-\frac{s}{n}\right)\|\mathbf{v}\|^{2}=0
$$

Note that $\mathbf{v}$ is a non-trivial geodesic vector field, we have $\|\mathbf{v}\|^{2} \neq 0$ and $M$ is connected. Consequently, above equation implies $\sigma=\frac{s}{n}$. Thus, Equations (12) and (13) imply

$$
A=\sigma I, \quad \varphi \mathbf{v}=0 .
$$

But Equation (14) gives

$$
(\nabla A)(X, Y)=X(\sigma) Y
$$

and therefore (5) implies

$$
R(X, Y) \mathbf{v}=X(\sigma) Y-Y(\sigma) X+(\nabla \varphi)(X, Y)-(\nabla \varphi)(Y, X)
$$

Taking now in above equation the inner product with $\mathbf{v}$, we derive

$$
X(\sigma) \alpha(Y)-Y(\sigma) \alpha(X)-g(Y,(\nabla \varphi)(X, \mathbf{v}))+g(X,(\nabla \varphi)(Y, \mathbf{v}))=0 .
$$

Replacing $Y$ with $\mathbf{v}$ in above equation and taking into account that $\varphi \mathbf{v}=0$ (Equation (14)) and $(\nabla \varphi)(\mathbf{v}, \mathbf{v})=0$ (Equation (4) with $A=\sigma I)$, we conclude

$$
X(\sigma)\|\mathbf{v}\|^{2}-\mathbf{v}(\sigma) \alpha(X)=g(\mathbf{v},(\nabla \varphi)(X, \mathbf{v}))=-g\left(\mathbf{v}, \varphi \nabla_{X} \mathbf{v}\right)=0 .
$$

Thus, we get

$$
\|\mathbf{v}\|^{2} \nabla \sigma=\mathbf{v}(\sigma) \mathbf{v},
$$

which, on taking the inner product with $\nabla \sigma$, yields

$$
\|\mathbf{v}\|^{2}\|\nabla \sigma\|^{2}=\mathbf{v}(\sigma)^{2}
$$

Now, we define a smooth function $h: M \rightarrow R$ by

$$
h=\frac{1}{2}\|\mathbf{v}\|^{2} .
$$

Then, using Equations (4) and (14), we obtain the gradient $\nabla h=\sigma \mathbf{v}$. We use Equation (4) and $A=\sigma I$, and compute the Hessian operator $A_{h}$,

$$
A_{h} X=X(\sigma) \mathbf{v}+\sigma^{2} X+\sigma \varphi X, \quad X \in \mathfrak{X}(M) .
$$

As the Hessian operator $A_{h}$ is symmetric, above equation implies

$$
2 \sigma \varphi X=\alpha(X) \nabla \sigma-X(\sigma) \mathbf{v},
$$


which gives

$$
4 \sigma^{2}\|\varphi\|^{2}=2\|\nabla \sigma\|^{2}\|\mathbf{v}\|^{2}-2 \mathbf{v}(\sigma)^{2} .
$$

Combining above equation with Equation (15), we arrive at $\sigma^{2}\|\varphi\|^{2}=0$ and as $\mathbf{v}$ is non-trivial, that is, $\sigma \neq 0$ on connected $M$, we have $\varphi=0$. Hence, Equation (4) takes the form

$$
\nabla_{X} \mathbf{v}=\sigma X,
$$

which proves that $\mathbf{v}$ is a concircular vector field.

Conversely, if $\mathbf{v}$ is concircular vector field, then we derive $A=\sigma I$ and $\varphi=0$ from Equation (4) and we have

$$
\sum_{i=1}^{n}(\nabla A)\left(e_{i}, e_{i}\right)=\nabla \sigma, s=\operatorname{Tr} A=n \sigma
$$

Thus, Equation (6) becomes

$$
\operatorname{Ric}(\mathbf{v}, \mathbf{v})=\mathbf{v}(\sigma)-\mathbf{v}(s)=-\frac{n-1}{n} \mathbf{v}(s)=\|\varphi\|^{2}-\frac{n-1}{n} \mathbf{v}(s),
$$

that is, the condition in the statement holds.

As a consequence of the above result, we derive:

Corollary 1. Suppose $(M, g)$ is a compact and connected Riemannian manifold of dimension $n$ and let $\mathbf{v}$ be a non-trivial closed geodesic vector field on $M$. Then, $\mathbf{v}$ is concircular if, and only if the Ricci curvature $\operatorname{Ric}(\mathbf{v}, \mathbf{v})$ satisfies

$$
\int_{M} \operatorname{Ric}(\mathbf{v}, \mathbf{v}) \geq \frac{n-1}{4 n} \int_{M}\left(\operatorname{Tr} £_{\mathbf{v}} g\right)^{2},
$$

where $£_{\mathbf{v}} g$ stands for the Lie derivative of $g$ with respect to $\mathbf{v}$.

Proof. Suppose that $\mathbf{v}$ is a closed geodesic vector field with $s=\operatorname{Tr} A$. Then, we have $\varphi=0$ and the given condition is

$$
\int_{M} \operatorname{Ric}(\mathbf{v}, \mathbf{v}) \geq \frac{n-1}{n} \int_{M} s^{2} .
$$

Now, using $\varphi=0$ in Equation (11), we deduce

$$
\int_{M}\left(\|A\|^{2}-\frac{1}{n} s^{2}\right)=\int_{M}\left(-\frac{n-1}{n} \mathbf{v}(s)-\operatorname{Ric}(\mathbf{v}, \mathbf{v})\right),
$$

which in view of

$$
\operatorname{div} s \mathbf{v}=\mathbf{v}(s)+s^{2},
$$

gives

$$
\int_{M}\left(\|A\|^{2}-\frac{1}{n} s^{2}\right)=\int_{M}\left(\frac{n-1}{n} s^{2}-\operatorname{Ric}(\mathbf{v}, \mathbf{v})\right) .
$$

Using inequality (17) in above equation, we derive

$$
\int_{M}\left(\|A\|^{2}-\frac{1}{n} s^{2}\right) \leq 0
$$

and following the steps of proof of Theorem 1 we conclude $A=\frac{s}{n} I$, that is, $\mathbf{v}$ is a concircular vector field. 
Conversely, if the vector field $\mathbf{v}$ is concircular, then $\nabla_{X} \mathbf{v}=\sigma X, X \in \mathfrak{X}(M)$. Moreover, we have

$$
R(X, Y) \mathbf{v}=X(\sigma) Y-Y(\sigma) X
$$

and we conclude

$$
\operatorname{Ric}(X, \mathbf{v})=-(n-1) X(\sigma),
$$

that is,

$$
\operatorname{Ric}(\mathbf{v}, \mathbf{v})=-(n-1) \mathbf{v}(\sigma)=-(n-1)\left(\operatorname{div} \sigma v-n \sigma^{2}\right) .
$$

Integrating this last equation and using $\left(\ell_{\mathbf{v}} g\right)(X, Y)=2 \sigma g(X, Y)$, we get

$$
\int_{M} \operatorname{Ric}(\mathbf{v}, \mathbf{v})=\frac{n-1}{4 n} \int_{M}\left(\operatorname{Tr} £_{\mathbf{v}} g\right)^{2} .
$$

Consequently, the required condition holds.

\section{Characterizing $n$-Spheres}

Let $\mathbf{S}^{n}(c)$ be the $n$-sphere of constant curvature $c>0$. Then, treating $\mathbf{S}^{n}(c)$ as hypersurface of the Euclidean space $\mathbf{E}^{n+1}=\left(R^{n+1},\langle\rangle,\right),\langle$,$\rangle being Euclidean metric, with unit normal vector field$ $N$, then the shape operator $A$ of the sphere is $A=-\sqrt{c} I$. Let us denote by $g$ the induced metric on $\mathbf{S}^{n}(c)$ and by $\nabla_{X}$ the covariant derivative with respect to the Riemannian connection on $\left(\mathbf{S}^{n}(c), g\right)$, $X \in \mathfrak{X}\left(\mathbf{S}^{n}(c)\right)$. Let $u^{1}, \ldots, u^{n+1}$ be the Euclidean coordinates on $\mathbf{E}^{n+1}$. The restriction of the coordinate vector field $\frac{\partial}{\partial u^{1}}$ to $\mathbf{S}^{n}(c)$ can be expressed as

$$
\frac{\partial}{\partial u^{1}}=\mathbf{u}+f N, \quad \mathbf{u} \in \mathfrak{X}\left(\mathbf{S}^{n}(c)\right), \quad f=\left\langle\frac{\partial}{\partial u^{1}}, N\right\rangle .
$$

Taking the covariant derivative with respect to $X \in \mathfrak{X}\left(\mathbf{S}^{n}(c)\right)$ and using fundamental equations of hypersurfaces, we derive

$$
0=\nabla_{X} \mathbf{u}-\sqrt{c} g(\mathbf{u}, X) N+X(f) N+\sqrt{c} f X .
$$

Equating tangential and normal components, we conclude

$$
\nabla_{X} \mathbf{u}=-\sqrt{c} f X, \quad X(f)=\sqrt{c} g(\mathbf{u}, X), \quad X \in \mathfrak{X}\left(\mathbf{S}^{n}(c)\right),
$$

which shows that $\mathbf{u}$ is a geodesic vector field on $\mathbf{S}^{n}(c)$ having the potential function $\sigma=-\sqrt{c} f$ and also that $\nabla f=\sqrt{c} \mathbf{u}$ holds. Moreover, the rough Laplacian $\Delta \mathbf{u}$ of the geodesic vector field $\mathbf{u}$ is given by

$$
\Delta \mathbf{u}=-\sqrt{c} \nabla f=-c \mathbf{u},
$$

that is, the geodesic vector field $\mathbf{u}$ is an eigenvector of the rough Laplacian operator on $\mathbf{S}^{n}(c)$.

Recall now that the energy $\mathbf{E}(\xi)$ of a vector field $\xi$ is given by

$$
\mathbf{E}(\xi)=\frac{1}{2} \int_{M}\|\xi\|^{2}
$$

We are able now to prove the following result that gives a characterization of $n$-sphere. 
Theorem 2. Let $\mathbf{v}$ be a non-trivial geodesic vector field on an $n$-dimensional compact and connected Riemannian manifold $(M, g)$ such that $\Delta \mathbf{v}=-\lambda \mathbf{v}$, for a constant $\lambda>0$. Then, $(M, g)$ is isometric to $n$-sphere $\mathbf{S}^{n}(\lambda)$ if and only if the energy $\mathbf{E}(\mathbf{v})$ satisfies

$$
\mathbf{E}(\mathbf{v}) \leq \frac{1}{8 n \lambda} \int_{M}\left(\operatorname{Tr} £_{\mathbf{v}} g\right)^{2}
$$

Proof. Suppose $\mathbf{v}$ is a non-trivial geodesic vector field such that $\Delta \mathbf{v}=-\lambda \mathbf{v}$ holds. Suppose the given condition holds, that is,

$$
\mathbf{E}(\mathbf{v}) \leq \frac{1}{2 n \lambda} \int_{M} s^{2}
$$

where $s=\operatorname{Tr} A$. Now, we remark that Equation (4) implies

$$
\Delta \mathbf{v}=\sum_{i=1}^{n}(\nabla A)\left(e_{i}, e_{i}\right)+\sum_{i=1}^{n}(\nabla \varphi)\left(e_{i}, e_{i}\right)=-\lambda \mathbf{v},
$$

which, on taking the inner product with $\mathbf{v}$, gives

$$
g\left(\mathbf{v}, \sum_{i=1}^{n}(\nabla A)\left(e_{i}, e_{i}\right)+\sum_{i=1}^{n}(\nabla \varphi)\left(e_{i}, e_{i}\right)\right)+\lambda\|\mathbf{v}\|^{2}=0
$$

Now, using Equations (8) and (9), it follows

$$
\operatorname{div}(A \mathbf{v}-\varphi \mathbf{v})=\|A\|^{2}+\|\varphi\|^{2}+g\left(\mathbf{v}, \sum_{i=1}^{n}(\nabla A)\left(e_{i}, e_{i}\right)+\sum_{i=1}^{n}(\nabla \varphi)\left(e_{i}, e_{i}\right)\right) .
$$

Inserting above equation in Equation (22) and integrating the resulting equation, we get

$$
\int_{M}\left(\|A\|^{2}+\|\varphi\|^{2}-\lambda\|\mathbf{v}\|^{2}\right)=0
$$

and we conclude

$$
\int_{M}\left(\left(\|A\|^{2}-\frac{1}{n} s^{2}\right)+\|\varphi\|^{2}\right)=2 \lambda\left(\mathbf{E}(\mathbf{v})-\frac{1}{2 \lambda n} \int_{M} s^{2}\right) .
$$

Using Schwartz's inequality and inequality (21) in above equation, we derive

$$
A=\frac{s}{n} I \text { and } \varphi=0
$$

Thus, using Equations (1), (4) and (23), we obtain $\sigma=\frac{s}{n}$ and Equation (4) takes the form

$$
\nabla_{X} \mathbf{v}=\sigma X, \quad X \in \mathfrak{X}(M) .
$$

Therefore, we conclude

$$
\Delta \mathbf{v}=\nabla \sigma=-\lambda \mathbf{v}
$$

Also, using Equation (24), we have divv $=n \sigma$, which on integration gives

$$
\int_{M} \sigma=0 \text {. }
$$


If $\sigma$ is a constant, above equation will imply $\sigma=0$ and this is a contradiction (since $\mathbf{v}$ is a non-trivial geodesic vector field). Thus $\sigma$ is a non-constant function and, by Equations (24) and (25), it satisfies

$$
\nabla_{X} \nabla \sigma=-\lambda \sigma X, \quad X \in \mathfrak{X}(M),
$$

which is Obata's differential equation for positive constant $\lambda$. Hence, $M$ is isometric to the sphere $\mathbf{S}^{n}(\lambda)$ (cf. [23] ). Conversely, suppose $M$ is isometric to the sphere $\mathbf{S}^{n}(\lambda)$. Then, by Equation (18), there is a geodesic vector field $\mathbf{u}$ on $\mathbf{S}^{n}(\lambda)$ induced by the coordinate vector field $\frac{\partial}{\partial u^{1}}$ on the Euclidean space $\mathbf{E}^{n+1}$, that satisfies $\Delta \mathbf{u}=-\lambda \mathbf{u}$. First, we shall verify that this geodesic vector field $\mathbf{u}$ with potential function $\sigma=-\sqrt{c} f$, where

$$
f=\left\langle\frac{\partial}{\partial u^{1}}, N\right\rangle
$$

is non-trivial, that is, $f \neq 0$. If $f=0$, then Equation (18) gives $\nabla_{X} \mathbf{u}=0, X \in \mathfrak{X}\left(\mathbf{S}^{n}(\lambda)\right)$ and as with $f=0$, the restriction of $\frac{\partial}{\partial u^{1}}=\mathbf{u}$, which on taking covariant derivative with respect to $X \in \mathfrak{X}\left(\mathbf{S}^{n}(\lambda)\right)$, gives $0=0-\sqrt{c} g(X, \mathbf{u})$ for all $X \in \mathfrak{X}\left(\mathbf{S}^{n}(\lambda)\right)$. This shows that $\mathbf{u}=0$, that is, $\frac{\partial}{\partial u^{1}}=0$ on $\mathbf{S}^{n}(\lambda)$, which is a contradiction (as $\frac{\partial}{\partial u^{1}}$ is a unit vector field). Hence, $\mathbf{u}$ is a non-trivial geodesic vector field. Note that by Equation (18), we have $\operatorname{div} \mathbf{u}=-n \sqrt{c} f$ and $\nabla f=\sqrt{c} \mathbf{u}$, which implies $\Delta f=-n c f$. Thus, we have

$$
\int_{M}\|\nabla f\|^{2}=n c \int_{M} f^{2} .
$$

Using $\mathbf{u}=\frac{1}{\sqrt{c}} \nabla f, \sigma=-\sqrt{c} f$ and above equation, we obtain that the energy of $\mathbf{u}$ is given by

$$
\mathbf{E}(\mathbf{u})=\frac{1}{2} \int_{M}\|\mathbf{u}\|^{2}=\frac{n}{2} \int_{M} \sigma^{2}=\frac{1}{8 n c} \int_{M}\left(\operatorname{Tr} £_{\mathbf{u}} g\right)^{2},
$$

where

$$
£_{\mathbf{u}} g=2 \sigma g
$$

Hence the required condition holds.

Also, we have the following

Theorem 3. Let $(M, g)$ be a compact and connected Riemannian manifold of dimension $n$ and constant scalar curvature $\tau$. Suppose $\mathbf{v}$ is a non-trivial closed geodesic vector field on $(M, g)$ with potential function $\sigma$ such that the Ricci curvature in the direction of the vector field $\nabla \sigma+c \mathbf{v}$ is non-negative for constant $c$, where $n(n-1) c=\tau$. Then, $c>0$ and $(M, g)$ is isometric to $n$-sphere $\mathbf{S}^{n}(c)$ if, and only if

$$
\int_{M} \operatorname{Ric}(\mathbf{v}, \mathbf{v}) \geq \frac{n-1}{4 n} \int_{M}\left(\operatorname{Tr} £_{\mathbf{v}} g\right)^{2} .
$$

Proof. Suppose $(M, g)$ is a compact and connected Riemannian manifold of dimension $n$ and constant scalar curvature $\tau=n(n-1) c$. We also suppose $\mathbf{v}$ is a non-trivial closed geodesic vector field such that Ric satisfies

$$
\operatorname{Ric}(\nabla \sigma+c \mathbf{v}, \nabla \sigma+c \mathbf{v}) \geq 0,
$$

where $\sigma$ denotes the potential function of $\mathbf{v}$, and also that the given condition holds, that is,

$$
\int_{M} \operatorname{Ric}(\mathbf{v}, \mathbf{v}) \geq \frac{n-1}{n} \int_{M} s^{2},
$$


where $s=\operatorname{Tr} A$. Then, by Corollary 1 , it follows that $\mathbf{v}$ is a concircular vector field, that is, $\nabla_{X} \mathbf{v}=\sigma X$, $X \in \mathfrak{X}(M)$. Consequently, we derive

$$
R(X, Y) \mathbf{v}=X(\sigma) Y-Y(\sigma) X
$$

and this implies

$$
\operatorname{Ric}(X, \mathbf{v})=-(n-1) X(\sigma)
$$

Thus, we have

$$
Q(\mathbf{v})=-(n-1) \nabla \sigma
$$

Taking divergence in (29) and also taking into account that $\tau$ is a constant and

$$
\sum_{i=1}^{n}(\nabla Q)\left(e_{i}, e_{i}\right)=0
$$

for a local orthonormal frame $\left\{e_{1}, \ldots, e_{n}\right\}$ on $M$, we conclude

$$
\Delta \sigma=-\frac{\tau}{n-1} \sigma=-n c \sigma
$$

We remark now that if $\sigma$ is a constant, then the integration of equation divv $=n \sigma$ will imply $\sigma=0$, which is a contradiction (since $\mathbf{v}$ is a non-trivial geodesic vector field). Hence $\sigma$ is non-constant function and Equation (30) implies that $\sigma$ is an eigenfunction of the Laplace operator $\Delta$ acting on smooth functions on compact $M$. This implies $c>0$ (for $c=0$ would again imply $\sigma$ is a constant, hence contradiction). Using now (30) and the Bochner formula

$$
\int_{M}\left(\operatorname{Ric}(\nabla \sigma, \nabla \sigma)+\left\|A_{\sigma}\right\|^{2}-(\Delta \sigma)^{2}\right)=0,
$$

we obtain

$$
\int_{M}\left(\operatorname{Ric}(\nabla \sigma, \nabla \sigma)+\left\|A_{\sigma}\right\|^{2}-n^{2} c^{2} \sigma^{2}\right)=0 .
$$

Using Equation (29), we derive

$$
\int_{M} \operatorname{Ric}(\nabla \sigma, \mathbf{v})=-(n-1) \int_{M}\|\nabla \sigma\|^{2}=-n(n-1) c \int_{M} \sigma^{2},
$$

where we have used Equation (30). Also, using Equation (18), we have

$$
\operatorname{Ric}(\mathbf{v}, \mathbf{v})=-(n-1) \mathbf{v}(\sigma)=-(n-1)\left(\operatorname{div} \sigma \mathbf{v}-n \sigma^{2}\right)
$$

which gives

$$
\int_{M} \operatorname{Ric}(\mathbf{v}, \mathbf{v})=n(n-1) \int_{M} \sigma^{2}
$$

Now, we have

$$
\operatorname{Ric}(\nabla \sigma+c \mathbf{v}, \nabla \sigma+c \mathbf{v})=\operatorname{Ric}(\nabla \sigma, \nabla \sigma)+2 c \operatorname{Ric}(\nabla \sigma, \mathbf{v})+c^{2} \operatorname{Ric}(\mathbf{v}, \mathbf{v})
$$


Integrating the above equation and using Equations (31)-(33), we get

$$
\begin{aligned}
\int_{M} \operatorname{Ric}(\nabla \sigma+c \mathbf{v}, \nabla \sigma+c \mathbf{v}) & =\int_{M}\left(-\left\|A_{\sigma}\right\|^{2}+n^{2} c^{2} \sigma^{2}-2 n(n-1) c^{2} \sigma^{2}+n(n-1) c^{2} \sigma^{2}\right) \\
& =\int_{M}\left(-\left\|A_{\sigma}\right\|^{2}+\frac{1}{n}(\Delta \sigma)^{2}-n c^{2} \sigma^{2}+n^{2} c^{2} \sigma^{2}-n(n-1) c^{2} \sigma^{2}\right),
\end{aligned}
$$

where we have used Equation (30). Thus, we have

$$
\int_{M}\left(\left\|A_{\sigma}\right\|^{2}-\frac{1}{n}(\Delta \sigma)^{2}\right)=-\int_{M} \operatorname{Ric}(\nabla \sigma+c \mathbf{v}, \nabla \sigma+c \mathbf{v}) .
$$

Using now inequality (27), we conclude

$$
\int_{M}\left(\left\|A_{\sigma}\right\|^{2}-\frac{1}{n}(\Delta \sigma)^{2}\right) \leq 0
$$

However, the Schwartz's inequality implies $\left\|A_{\sigma}\right\|^{2} \geq \frac{1}{n}(\Delta \sigma)^{2}$ and in view of inequality (34), we conclude

$$
\left\|A_{\sigma}\right\|^{2}=\frac{1}{n}(\Delta \sigma)^{2}
$$

The above equality holds if, and only if $A_{\sigma}=\frac{\Delta \sigma}{n} I$, that is, using Equation (30)

$$
\nabla_{X} \nabla \sigma=-c \sigma X, \quad X \in \mathfrak{X}(M),
$$

where $\sigma$ is a non-constant function. However, the above equation is Obata's differential equation that implies $(M, g)$ is isometric to the sphere $\mathbf{S}^{n}(c)$.

Conversely, suppose $(M, g)$ is isometric to the sphere $\mathbf{S}^{n}(c)$. Then, it is known that $\mathbf{S}^{n}(c)$ admits a non-trivial closed geodesic vector field $\mathbf{u}$ satisfying Equation (18) with potential function $\sigma=-\sqrt{c} f$, where $\nabla f=\sqrt{c} \mathbf{u}$. This gives $\nabla \sigma+c \mathbf{u}=0$, that is, the following relation holds

$$
\operatorname{Ric}(\nabla \sigma+c \mathbf{u}, \nabla \sigma+c \mathbf{u})=0 .
$$

Moreover,

$$
\operatorname{Ric}(\mathbf{u}, \mathbf{u})=(n-1) c\|\mathbf{u}\|^{2}=(n-1)\|\nabla f\|^{2}
$$

and $\Delta f=-n c f$. Thus, we get

$$
\int_{M} \operatorname{Ric}(\mathbf{u}, \mathbf{u})=(n-1) \int_{M}\|\nabla f\|^{2}=n(n-1) c \int_{M} f^{2}=n(n-1) \int_{M} \sigma^{2} .
$$

Also, we have $\left.\left(£_{\mathbf{u}} g\right)(X, Y)=2 \sigma g(X, Y)\right)$, that is $\operatorname{Tr}\left(£_{\mathbf{u}} g\right)=2 n \sigma$. Hence, using Equation (35), we derive

$$
\int_{M} \operatorname{Ric}(\mathbf{u}, \mathbf{u})=\frac{n-1}{4 n} \int_{M}\left(\operatorname{Tr}\left(£_{\mathbf{u}} g\right)\right)^{2},
$$

that is, all the requirements are met.

Remark 1. Another characterization of the spheres in terms of the existence of non-trivial geodesic vector fields satisfying a certain inequality was recently obtained in ([31] Theorem 2), but with different hypotheses-namely, $(M, g)$ is a compact and connected Riemannian manifold of positive Ricci curvature and constant scalar curvature. Note that the inequality in ([31] Theorem 2) reduces to the inequality in the statement of the above 
theorem, provided that the non-trivial geodesic vector field is closed, but Theorem 3 is obtained in a more general setting, namely, $(M, g)$ is a compact and connected Riemannian manifold of constant scalar curvature $\tau$, such that the Ricci curvature is non-negative only in the direction of a particular vector field. It has been shown that this condition is strong enough to imply $\tau>0$, and this is one of the key ingredients in the proof.

\section{A Characterization of Euclidean Spaces}

Consider the Euclidean space $\mathbf{E}^{n}$ and the position vector field

$$
\mathbf{v}=\sum_{i=1}^{n} u^{i} \frac{\partial}{\partial u^{i}}
$$

Then, $\mathbf{v}$ satisfies

$$
\nabla_{\mathbf{v}} \mathbf{v}=\mathbf{v}
$$

that is, $\mathbf{v}$ is geodesic, having the potential function $\sigma=1$. Moreover, if we compute the rough Laplacian $\Delta \mathbf{v}$ of this geodesic vector field $\mathbf{v}$ on the Euclidean space $\mathbf{E}^{n}$, we see that $\Delta \mathbf{v}=0$, that is, this geodesic vector field is harmonic. This raises an interesting question: Is it an n-dimensional complete and connected Riemannian manifold $(M, g)$ admitting a harmonic geodesic vector field, necessarily isometric to $\mathbf{E}^{n}$ ? In the following, we partially answer this question, finding a characterization of $\mathbf{E}^{n}$.

Theorem 4. Let $(M, g)$ be a complete and connected Riemannian manifold of dimension $n$. Then, $(M, g)$ is isometric to the Euclidean space $\mathbf{E}^{n}$ if, and only if it admits a non-trivial harmonic geodesic vector field $\mathbf{v}$, such that the length function

$$
h=\frac{1}{2}\|\mathbf{v}\|^{2}
$$

satisfies

$$
\Delta h \leq \frac{1}{4 n}\left(\operatorname{Tr} £_{\mathbf{v}} g\right)^{2}
$$

Proof. Suppose $(M, g)$ admits a non-trivial geodesic vector field $\mathbf{v}$ satisfying $\Delta \mathbf{v}=0$, and the smooth function $h$ satisfies the given condition

$$
\Delta h \leq \frac{1}{n} s^{2},
$$

where $s=\operatorname{Tr} A$. Using Equation (4), we have

$$
\nabla h=A \mathbf{v}-\varphi \mathbf{v},
$$

which, on using Equations (8) and (9), gives

$$
\Delta h=\|A\|^{2}+\|\varphi\|^{2}+g\left(\mathbf{v}, \sum_{i=1}^{n}\left((\nabla A)\left(e_{i}, e_{i}\right)+(\nabla \varphi)\left(e_{i}, e_{i}\right)\right)\right) .
$$

Now, using Equation (4), we find that the rough Laplacian $\Delta \mathbf{v}$ of the geodesic vector field is given by

$$
\Delta \mathbf{v}=\sum_{i=1}^{n}\left((\nabla A)\left(e_{i}, e_{i}\right)+(\nabla \varphi)\left(e_{i}, e_{i}\right)\right) .
$$

Thus, for the harmonic geodesic vector field $\mathbf{v}$, Equation (38) becomes

$$
\Delta h=\|A\|^{2}+\|\varphi\|^{2},
$$

that is,

$$
\Delta h-\frac{1}{n} s^{2}=\left(\|A\|^{2}-\frac{1}{n} s^{2}\right)+\|\varphi\|^{2} .
$$


Using the above equation, in view of Schwartz's inequality and inequality (36), we conclude

$$
A=\frac{s}{n} I, \quad \varphi=0, \quad \Delta h=\frac{1}{n} s^{2} .
$$

However, first, Equation in (40) gives

$$
\sum_{i=1}^{n}(\nabla A)\left(e_{i}, e_{i}\right)=\frac{1}{n} \nabla s,
$$

while Equation (39), with $\varphi=0$ and harmonic $\mathbf{v}$, gives

$$
\sum_{i=1}^{n}(\nabla A)\left(e_{i}, e_{i}\right)=0 .
$$

Thus, Equation (41) implies $s$ is a constant, and using Equations (40) and (4), we derive that $\sigma=\frac{s}{n}$ is a constant, say $c$. Note that $\mathbf{v}$ being a non-trivial geodesic vector field, we have the constant $c \neq 0$. Now, in view of Equation (40), we conclude that Equation (37) takes the form

$$
\nabla h=c \mathbf{v}
$$

and it follows from the above equation that the function $h$ is not a constant, as the constant $c \neq 0$ and $\mathbf{v} \neq 0$ is a non-trivial geodesic vector field. Now, as the Equation (4) changes to

$$
\nabla_{X} \mathbf{v}=c X, \quad X \in \mathfrak{X}(M),
$$

by using the above equation in the defining equation of the Hessian operator $A_{h}$, the following expression for $A_{h}$ is obtained:

$$
A_{h} X=c^{2} X, \quad X \in \mathfrak{X}(M) .
$$

Thus, we have Hess $(h)=c^{2} g$, and this shows that $M$ is isometric to $\mathbf{E}^{n}$ (cf. [34] Theorem 1).

Conversely, suppose $M$ is isometric to the Euclidean space $\mathbf{E}^{n}$. Then, as we have seen before, the position vector field

$$
\mathbf{v}=\sum_{i=1}^{n} u^{i} \frac{\partial}{\partial u^{i}}
$$

is harmonic and $h=\frac{1}{2}\|\mathbf{v}\|^{2}$ satisfies $\Delta h=n$. Moreover, $A=I$ and thus, $\operatorname{Tr}\left(\ell_{\mathbf{v}} g\right)=2 n$, where $g$ is the Euclidean metric. Hence, the following relation holds

$$
\Delta h=\frac{1}{4 n}\left(\operatorname{Tr} £_{\mathbf{v}} g\right)^{2},
$$

and the conclusion is clear.

Remark 2. A distinct characterization of the Euclidean spaces in terms of the existence of non-trivial geodesic vector fields satisfying a certain inequality was recently obtained in ([31] Theorem 1), but the inequality in the statement is of a different nature, involving the Ricci curvature in the direction of the geodesic vector field, as well as the potential function of this vector field.

Author Contributions: Conceptualization and methodology, S.D.; formal analysis, G.-E.V.; writing original draft preparation, S.D.; writing—review and editing, G.-E.V.; supervision, J.M.; project administration, N.B.T.; and funding acquisition, N.B.T. All authors have read and agreed to the published version of the manuscript.

Funding: The authors extend their appreciations to the Deanship of Scientific Research King Saud University for funding this work through research group no (RG-1440-142). J.M. was supported by a grant of Palacky University Olomouc (grant IGA no. 2020014 "Mathematical Structures"). 
Acknowledgments: The authors would like to express sincere appreciation to the anonymous reviewers for their valuable comments and suggestions which helped to improve the manuscript.

Conflicts of Interest: The authors declare no conflicts of interest.

\section{References}

1. Chen, B.-Y.; Nagano, T. Harmonic metrics, harmonic tensors and Gauss maps. J. Math. Soc. Jpn. 1984, 36, 295-313. [CrossRef]

2. Sharma, R. Some results on almost Ricci solitons and geodesic vector fields. Beitr. Algebra Geom. 2018, 59, 289-294. [CrossRef]

3. Yano, K.; Nagano, T. On geodesic vector fields in a compact orientable Riemannian space. Comment. Math. Helv. 1961, 35, 55-64. [CrossRef]

4. Chen, B.-Y.; Deshmukh, S.; Ishan, A.A. On Jacobi-type vector fields on Riemannian manifolds. Mathematics 2019, 7, 1139. [CrossRef]

5. Deshmukh, S. Jacobi-type vector fields and Ricci solitons. Bull. Math. Soc. Sci. Math. Roum. 2012, 55, 41-50.

6. Chen, B.-Y. Some results on concircular vector fields and their applications to Ricci solitons. Bull. Korean Math. Soc. 2015, 52, 1535-1547. [CrossRef]

7. Fialkow, A. Conformal geodesics. Trans. Am. Math. Soc. 1939, 45, 443-473. [CrossRef]

8. Mosayebi, P.; Kobzas, D.; Murtha, A.; Jagersand, M. Tumor invasion margin on Riemannian space of brain fibers. Med. Image Anal. 2012, 16, 361-373. [CrossRef]

9. Takeno, H. Concircular scalar field in spherically symmetric space-times I. Tensor (N. S.) 1967, 20, 167-176.

10. Chen, B.-Y.; Verstraelen, L. A link between torse-forming vector fields and rotational hypersurfaces. Int. J. Geom. Methods Mod. Phys. 2017, 14, 1750177. [CrossRef]

11. Mantica, C.A.; Molinari, L.G. Twisted Lorentzian manifolds: A characterization with torse-forming time-like unit vectors. Gen. Relativ. Gravit. 2017, 49, 51. [CrossRef]

12. Mihai, A.; Mihai, I. Torse forming vector fields and exterior concurrent vector fields on Riemannian manifolds and applications. J. Geom. Phys. 2013, 73, 200-208. [CrossRef]

13. Yano, K. On torse forming direction in a Riemannian space. Proc. Imp. Acad. Tokyo 1944, 20, 340-346. [CrossRef]

14. Berestovskii, V.; Nikonorov, Y. Killing vector fields of constant length on Riemannian manifolds. Sib. Math. J. 2008, 49, 395-407. [CrossRef]

15. Flores, J.L.; Javaloyes, M.A.; Piccione, P. Periodic geodesics and geometry of compact Lorentzian manifolds with a Killing vector field. Math. Z. 2011, 267, 221-233. [CrossRef]

16. De la Fuente, D. The geodesic completeness of compact Lorentzian manifolds admitting a timelike Killing vector field revisited: Two new proofs. Publ. Inst. Math. (Beograd) (N. S.) 2018, 104, 121-125.

17. Lynge, W.C. Sufficient conditions for periodicity of a Killing vector field. Proc. Am. Math. Soc. 1973, 38, 614-616. [CrossRef]

18. Rong, X. Positive curvature, local and global symmetry, and fundamental groups. Am. J. Math. 1999, 121, 931-943. [CrossRef]

19. Yorozu, S. Killing vector fields on complete Riemannian manifolds. Proc. Am. Math. Soc. 1982, 84, 115-120. [CrossRef]

20. Yorozu, S. Killing vector fields on noncompact Riemannian manifolds with boundary. Kodai Math. J. 1982, 5, 426-433. [CrossRef]

21. Alías, L.J.; de Lira, J.H.; Rigoli, M. Mean curvature flow solitons in the presence of conformal vector fields. J. Geom. Anal. 2020, 30, 1466-1529. [CrossRef]

22. Diógenes, R.; Ribeiro, E., Jr.; Silva Filho, J. Gradient Ricci solitons admitting a closed conformal vector field. J. Math. Anal. Appl. 2017, 455, 1975-1983. [CrossRef]

23. Obata, M. Conformal transformations of Riemannian manifolds. J. Diff. Geom. 1970, 4, 311-333. [CrossRef]

24. Obata, M. The conjectures about conformal transformations. J. Diff. Geom. 1971, 6, 247-258. [CrossRef]

25. Brinkmann, H.W. Einstein spaces which mapped conformally on each other. Math. Ann. 1925, 94, 119-145. [CrossRef]

26. Mikes, J.; Stepanova, E.; Vanzurova, E. Differential Geometry of Special Mappings; Palacký University Press: Olomouc, Czech Republic, 2015. 
27. Hinterleitner, I. Holomorphically projective mappings of (pseudo-) Kähler manifolds preserve the class of differentiability. Filomat 2016, 30, 3115-3122. [CrossRef]

28. Mikeš, J. Holomorphically projective mappings and their generalizations. J. Math. Sci. (N. Y.) 1998, 89, 1334-1353. [CrossRef]

29. Petrović, M.Z.; Velimirović, L.S. Generalized almost Hermitian spaces and holomorphically projective mappings. Mediterr. J. Math. 2020, 17, 74. [CrossRef]

30. Deshmukh, S.; Khan, V.A. Geodesic vector fields and Eikonal equation on a Riemannian manifold. Indag. Math. 2019, 30, 542-552. [CrossRef]

31. Deshmukh, S.; Peska, P.; Turki, N.B. Geodesic vector fields on a Riemannian manifold. Mathematics 2020, 8, 137. [CrossRef]

32. Besse, A.L. Einstein Manifolds; Springer: London, UK, 1987.

33. O'Neill, B. Semi-Riemannian Geometry with Applications to Relativity; Academic Press: London, UK, 1983.

34. Pigola, S.; Rimoldi, M.; Setti, A.G. Remarks on non-compact gradient Ricci solitons. Math. Z. 2011, 268, 777-790. [CrossRef]

(c) 2020 by the authors. Licensee MDPI, Basel, Switzerland. This article is an open access article distributed under the terms and conditions of the Creative Commons Attribution (CC BY) license (http:/ / creativecommons.org/licenses/by/4.0/). 